PROCEEDINGS OF THE

AMERICAN MATHEMATICAL SOCIETY

Volume 132, Number 10, Pages 3135-3136

S 0002-9939(04)07597-5

Article electronically published on June 2, 2004

\title{
CORRIGENDUM TO \\ "EXISTENCE THEORY FOR FIRST ORDER DISCONTINUOUS FUNCTIONAL DIFFERENTIAL EQUATIONS"
}

\author{
JOSÉ ÁNGEL CID, EDUARDO LIZ, AND RODRIGO L. POUSO \\ (Communicated by Carmen C. Chicone)
}

The main result in [3], Theorem 3.3, is incorrect. We will present a counterexample and a repair.

To construct a counterexample, we use a result of Davis [1]: a necessary and sufficient condition for a lattice to be complete is that every nondecreasing function from the lattice into itself has a fixed point.

For $r>0$, let $\mathcal{L}^{\infty}([-r, 0])$ be the set of all bounded Lebesgue-measurable real functions on $[-r, 0]$, and let $\bar{\alpha}, \bar{\beta} \in \mathcal{L}^{\infty}([-r, 0])$ be given by $\bar{\alpha} \equiv 0$ and $\bar{\beta} \equiv 1$. The functional interval $[\bar{\alpha}, \bar{\beta}]=\left\{\gamma \in \mathcal{L}^{\infty}([-r, 0]): \bar{\alpha} \leq \gamma \leq \bar{\beta}\right\}$ is an incomplete lattice. Thus, by Davis' result, there exists a nondecreasing mapping $A:[\bar{\alpha}, \bar{\beta}] \rightarrow[\bar{\alpha}, \bar{\beta}]$ with no fixed point.

The problem

$$
\left\{\begin{array}{l}
x^{\prime}(t)=0, \quad \text { for a.a. } t \in[0,1], \\
x(\theta)=A x_{\mid[-r, 0]}(\theta), \quad \text { for all } \theta \in[-r, 0],
\end{array}\right.
$$

fits the assumptions required in Theorem 3.3 with the lower and upper solutions $\alpha \equiv 0$ and $\beta \equiv 1$ on $[-r, 1]$, and the mapping

$$
B: \xi \in \mathcal{S}_{r}^{1} \mapsto B \xi:=A\left(\sup \left\{\bar{\alpha}, \inf \left\{\xi_{\mid[-r, 0]}, \bar{\beta}\right\}\right\}\right) \in \mathcal{L}^{\infty}([-r, 0]) .
$$

Notice that $B \xi=A \xi_{\mid[-r, 0]}$ whenever $\alpha \leq \xi \leq \beta$. But, contrary to the statement of Theorem 3.3, (0.1) has no solution between $\alpha$ and $\beta$ in the sense of Definition 3.2, i.e., a solution $x:[-r, 1] \rightarrow \mathbb{R}$ with $x_{\mid[-r, 0]} \in \mathcal{L}^{\infty}([-r, 0])$, because the restriction of such a solution to $[-r, 0]$ would be a fixed point for $A$, a contradiction.

The analogue of Theorem 3.3 with $\mathcal{L}^{\infty}([-r, 0])$ replaced by $\mathcal{B}([-r, 0])$, the set of all bounded real functions on $[-r, 0]$, is true.

The proof remains the same as in [3] except that Claim 2 must be replaced by

Claim $2^{\prime}$. If $C$ is a chain in $[\alpha, \beta]$, then $\sup G[C]$ and $\inf G[C]$ exist in $[\alpha, \beta]$. In particular, $\sup G[C]$ and $\inf G[\bar{C}]$ exist in $\mathcal{S}_{r}^{T}$ when $C$ is the well-ordered chain of $G$-iterations of $\alpha$, and $\bar{C}$ is the inversely well-ordered chain of $G$-iterations of $\beta$.

Claim $2^{\prime}$ follows from Theorem 1.2.3 in [2], included as reference [7] in [3].

The corrected version of Theorem 3.3, which is obtained by replacing $\mathcal{L}^{\infty}([-r, 0])$ with $\mathcal{B}([-r, 0])$, does not change the conclusions in Examples 4.1 and 4.2 in 3$]$.

Received by the editors November 12, 2003 and, in revised form, December 10, 2003.

2000 Mathematics Subject Classification. Primary 34A12, 34K07, 34K10.

(C)2004 American Mathematical Society 


\section{REFERENCES}

[1] Anne C. Davis, A characterization of complete lattices, Pacific J. Math. 5 (1955), 311-319. $\operatorname{MR} 17: 574 \mathrm{e}$

[2] S. Heikkilä and V. Lakshmikantham, "Monotone iterative techniques for discontinuous nonlinear differential equations", Marcel Dekker, New York (1994). MR 95d:34002

[3] Eduardo Liz and Rodrigo L. Pouso, Existence theory for first order discontinuous functional differential equations, Proc. Amer. Math. Soc. 130, 11 (2002), 3301-3311. MR 2004d:34012

Departamento de Análise Matemática, Facultade de Matemáticas, Campus Sur, Universidade de Santiago de Compostela, 15782 Santiago de Compostela, Spain

E-mail address: angelcid@usc.es

Departamento de Matemática Aplicada, E.T.S.I. Telecomunicación, Universidad de Vigo, Campus Marcosende, 36280 Vigo, Spain

E-mail address: eliz@dma.uvigo.es

Departamento de Análise Matemática, Facultade de Matemáticas, Campus Sur, Universidade de Santiago de Compostela, 15782 Santiago de Compostela, Spain

E-mail address: rodrigolp@usc.es 